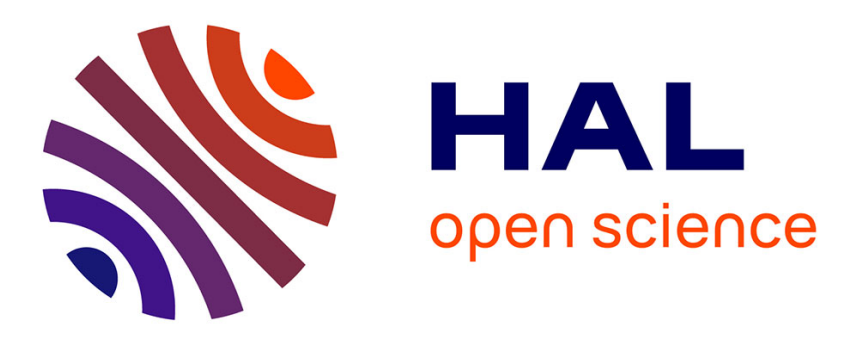

\title{
Hybrid constitutive modeling: data-driven learning of corrections to plasticity models
}

Rubén Ibáñez, Emmanuelle Abisset-Chavanne, David González, Jean Louis Duval, Elias Cueto, Francisco Chinesta

\section{- To cite this version:}

Rubén Ibáñez, Emmanuelle Abisset-Chavanne, David González, Jean Louis Duval, Elias Cueto, et al.. Hybrid constitutive modeling: data-driven learning of corrections to plasticity models. International Journal of Material Forming, 2019, 12 (4), pp.717-725. 10.1007/s12289-018-1448-x . hal-02375057

\section{HAL Id: hal-02375057 https://hal.science/hal-02375057}

Submitted on 21 Nov 2019

HAL is a multi-disciplinary open access archive for the deposit and dissemination of scientific research documents, whether they are published or not. The documents may come from teaching and research institutions in France or abroad, or from public or private research centers.
L'archive ouverte pluridisciplinaire HAL, est destinée au dépôt et à la diffusion de documents scientifiques de niveau recherche, publiés ou non, émanant des établissements d'enseignement et de recherche français ou étrangers, des laboratoires publics ou privés. 


\title{
Hybrid constitutive modeling: data-driven learning of corrections to plasticity models
}

\author{
Rubén Ibáñez ${ }^{1}$ - Emmanuelle Abisset-Chavanne ${ }^{1} \cdot$ David González $^{2} \cdot$ Jean-Louis Duval ${ }^{3}$. Elias Cueto ${ }^{2}$. \\ Francisco Chinesta ${ }^{4}$ (D)
}

\begin{abstract}
In recent times a growing interest has arose on the development of data-driven techniques to avoid the employ of phenomenological constitutive models. While it is true that, in general, data do not fit perfectly to existing models, and present deviations from the most popular ones, we believe that this does not justify (or, at least, not always) to abandon completely all the acquired knowledge on the constitutive characterization of materials. Instead, what we propose here is, by means of machine learning techniques, to develop correction to those popular models so as to minimize the errors in constitutive modeling.
\end{abstract}

Keywords Machine learning $\cdot$ Data-driven computational mechanics $\cdot$ Plasticity $\cdot$ Model learning

\section{Introduction}

Plenty of effort has been dedicated throughout history to create very accurate models. As an example, the reader may think about all different models formulated, for instance,

Francisco Chinesta

Francisco.Chinesta@ensam.eu

Rubén Ibáñez

Ruben.Ibanez-Pinillo@eleves.ec-nantes.fr

Emmanuelle Abisset-Chavanne

Emmanuelle.Abisset-Chavanne@ec-nantes.fr

Jean-Louis Duval

jean-louis.duval@esi-group.com

Elias Cueto

ecueto@unizar.es

1 ESI Chair, ENSAM ParisTech, 151, bvd. de 1'Hôpital, 75013 Paris, France

2 Aragon Institute of Engineering Research, Universidad de Zaragoza, Maria de la Luna s/n, 50018 Zaragoza, Spain

3 ESI Group, 99 rue des Solets, 94150 Rungis, France

4 PIMM - Procédés et Ingénierie en Mécanique et Matériaux, ENSAM ParisTech, 151 boulevard de l'Hôpital, 75013 Paris, France in hyperelasticity. These include the classical Saint-Venant, Neo-Hookean, Ogden [1], Arruda and Boyce [2], Holzapfel and Gasser [3], to name but a few. Another field in which plenty of constitutive models have been developed is plasticity, where we can highlight the classical models by Tresca, Von Misses [4] or Hill [5], among others.

However, we also know that no model is perfect: it is always subjected to certain limiting hypothesis, experimental noise, etc. Indeed, even if you could calibrate a model perfectly well, no guarantee is given that for another set of experiments, different from the calibration ones, the model is going to provide you a perfect result.

It has been argued that constitutive models are of a lower epistemic level than other, more fundamental, equations. This last group includes equilibrium and compatibility, for instance. This reasoning is at the origin of the so-called data-driven computational mechanics approach. In essence, this approach tries to substitute phenomenological, always imperfect models by experimental data. These techniques employ a variety of methods to determine, in essence, the stress tensor corresponding to a given strain state. Thus, for instance, works by M. Ortiz and coworkers employ nearest neighbor interpolation [6, 7], while in [8-12] the authors employ different manifold techniques to define the constitutive manifold of a given material, i.e., a low-dimensional representation of the constitutive equation based solely on data. Liu and coworkers [13] employ clustering techniques, while Montans et al. employ 
spline approximation to the strain density functional in a hyperelastic context $[14,15]$.

These approaches can be embedded in an even more general context. Several works have been devoted to unveil governing equations from data [16-18]. These may include laws in the form of partial differential equations, for instance [19-21].

The main aim of this work is to provide an alternative route by enhancing or correcting existing, well-known, models with information coming from data, thus performing a sort of data-driven correction. In this first work a special effort is put on the correction of plastic yield functions, while work in progress adresses more complex scenarios involving hardening and damage.

The proposed data driven correction technique is conceptually simple. Imagine that our departure point is a given, well-known parametric model $\mathcal{M}(\boldsymbol{p})$. It is important to keep in mind that we are looking for an enhancement or correction of the previous model based on the available experimental results. Therefore, a discrepancy model $\mathcal{D}(\boldsymbol{c})$, which applies to the first model, needs to be defined. So to speak, reality, $\mathcal{R}$, is approximated as

$\mathcal{R}=\mathcal{M}(\boldsymbol{p})+\left.\mathcal{D}(\boldsymbol{c})\right|_{\boldsymbol{p}}$,

where $\boldsymbol{p}$ represents the set of parameters governing the model and $c$ represents the set of parameters needed to define the necessary correction.

Since our measurement capabilities will in general be constrained to some experimentally observable quantities, both our objective reality and the correction to the model will be restricted to these experimental settings. In other words,

$\left.\mathcal{R}\right|_{s} \approx \mathcal{M}(\boldsymbol{p})+\left.\mathcal{D}(\boldsymbol{c})\right|_{p, s}$.

It is worth to mention that the way we define the observables $s$ could have an important impact over the calibration of the set of correction parameters, $\boldsymbol{c}$. We assume that a set of experiences is defined such that the entire parametric space $\boldsymbol{c}$ could be determined.

The outline of the paper is as follows. In Section "Problem statement" we present the developed methodology with the help of a toy problem. Section "Reconstruction of the error response surface by sparse sampling" introduces a sparse sampling technique able to describe the error surface with a minimum number of control points. In Section "Numerical results" the numerical results are presented, showing the performance of the proposed methodology.

\section{Problem statement}

In the present work, we will try to capture the plastic yield function of a particular material with the help of a well known plasticity model and try to develop the necessary corrections based on data. Recall that a plastic yield function can be seen as a hypersurface living in the stress space, $\sigma \in$ $\mathbb{R}^{6}$. Typically, this surface is parameterized using a finite set of parameters $(\boldsymbol{p})$ given by the physics-based model, $\mathcal{M}(\boldsymbol{p})$. Moreover, it will depend also on the correction or discrepancy model $\mathcal{D}(\boldsymbol{c})$. Therefore, our reality $\mathcal{R}$, in the
Fig. 1 Different views of Barlat Yld2004-18p plastic yield function. Color map represents the value of $\tau_{x y}$

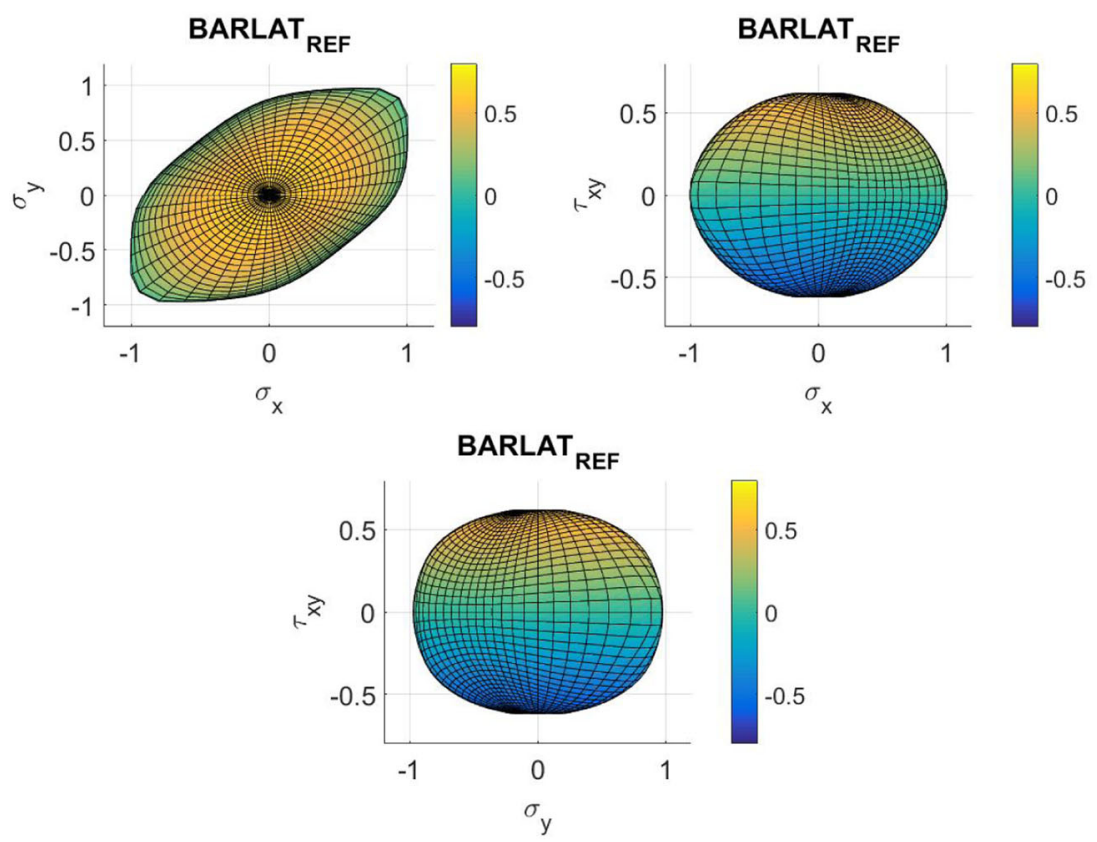


form of a general, yet unknown, plastic yield function, can be written as

$\mathcal{R}=\mathcal{F}_{Y}(\boldsymbol{\sigma} ; \boldsymbol{p}, \boldsymbol{c})=0$.

For the sake of simplicity and ease of representation, but without loosing generality, we will constraint ourselves to the plane stress hypothesis. Therefore, our plastic yield function is defined in a three dimensional space corresponding to the three active stress components, $\sigma_{x x}$, $\sigma_{y y}$, and $\tau_{x y}$. This three-dimensional stress state is easily expressed in spherical coordinates as

$\sigma_{x x}=R(\alpha, \beta) \cos (\alpha) \sin (\beta)$,

$\sigma_{y y}=R(\alpha, \beta) \sin (\alpha) \sin (\beta)$,

$\tau_{x y}=R(\alpha, \beta) \cos (\beta)$,

since the plastic yield function is often a convex closed surface. Here, $R(\alpha, \beta)$ defines the radius in spherical coordinates for any possible angle. Therefore, a parameterization $R(\alpha, \beta ; \boldsymbol{p})$ directly determines the shape of $\mathcal{F}_{Y}$.

Let us assume that reality, $\mathcal{R}$, is perfectly described by a Barlat Yld2004-18p yield function [22, 23], shown in Fig. 1, so that $\mathcal{R}=\mathcal{F}_{Y}^{B}$. Assume that this model has never been defined, so that we need to approximate reality - known indirectly through experimental results - by employing a well-known, yet inexact model. For this purpose we have chosen a quadratic Hill plastic yield function

$$
\begin{array}{r}
\mathcal{M}(\boldsymbol{p})=\mathcal{F}_{Y}^{H}\left(\sigma_{x x}, \sigma_{y y}, \tau_{x y} ; F, G, H, N\right) \\
=F \sigma_{y y}^{2}+G \sigma_{x x}^{2}+H\left(\sigma_{x x}-\sigma_{y y}\right)^{2}+N \tau_{x y}^{2}-\sigma_{0}^{2} .
\end{array}
$$

As it can be noticed, this yield criterion presents a parameterization based on four coefficients, i.e., $|\boldsymbol{p}|=4$ and $\boldsymbol{p}=\{F, G, H, N\}$.

Both Hill's and Barlat's models have been chosen to represent a well-known model that does not fit exactly to reality, and to govern the reality, respectively. This choice purely arbitrary, and its sole purpose is to show that a model can effectively be corrected so as to fit experimental evidence.

Figure 2 depicts the shape of a quadratic Hill yield criterion with $F=2.1, G=1.8, H=0.7$, and $N=1.9$. As it can be noticed, convexity is fulfilled and it defines a smooth closed surface in the stress space.

The discrepancy model $\mathcal{D}(\boldsymbol{c})$ is assumed to provide a correction to the Hill model so as to satisfy the Barlat Yld2004-18p model, from which the (synthetic) experimental data were obtained. To construct this discrepancy model, in the absence of any knowledge about the sought Barlat model, we chose to employ an as general as possible parameterization. We defined a set of eight control points distributed along the plane $\tau_{x y}=0$-corresponding to a biaxial experiment-, plus another degree of freedom relative to the maximum shear points defined along the line $\sigma_{x x}=0$, $\sigma_{y y}=0$-thus giving a pure shear experiment-. Hence, $|\boldsymbol{c}|=9$. Obviously, more control points could be added, if more precision is sought. The method does not present any limitation in this sense.

Interpolation between all degrees of freedom is done by means of natural neighbor interpolation, which provides $\mathcal{C}^{1}$. continuous shape functions, except from the data points, where it is simply continuous [24]. Figure 3 depicts the
Fig. 2 Different views of the quadratic Hill plastic yield function. Color map represents the value of $\tau_{x y} . F=2.1$, $G=1.8, H=0.7$ and $N=1.9$ were taken
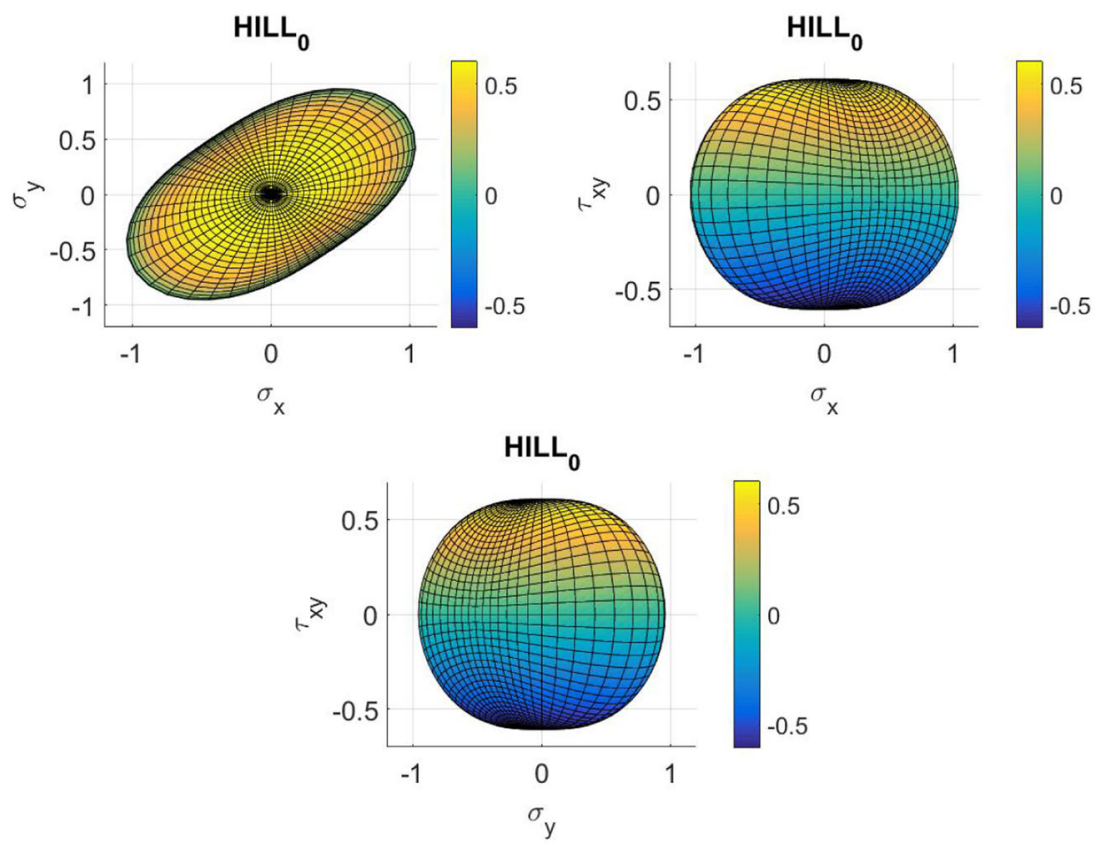
Fig. 3 Perturbed quadratic Hill plastic yield function by moving only one degree of freedom of $\mathcal{D}(\boldsymbol{c})$. Color map represents the magnitude of the perturbation. $F=2.1, G=1.8, H=0.7$, and $N=1.9$
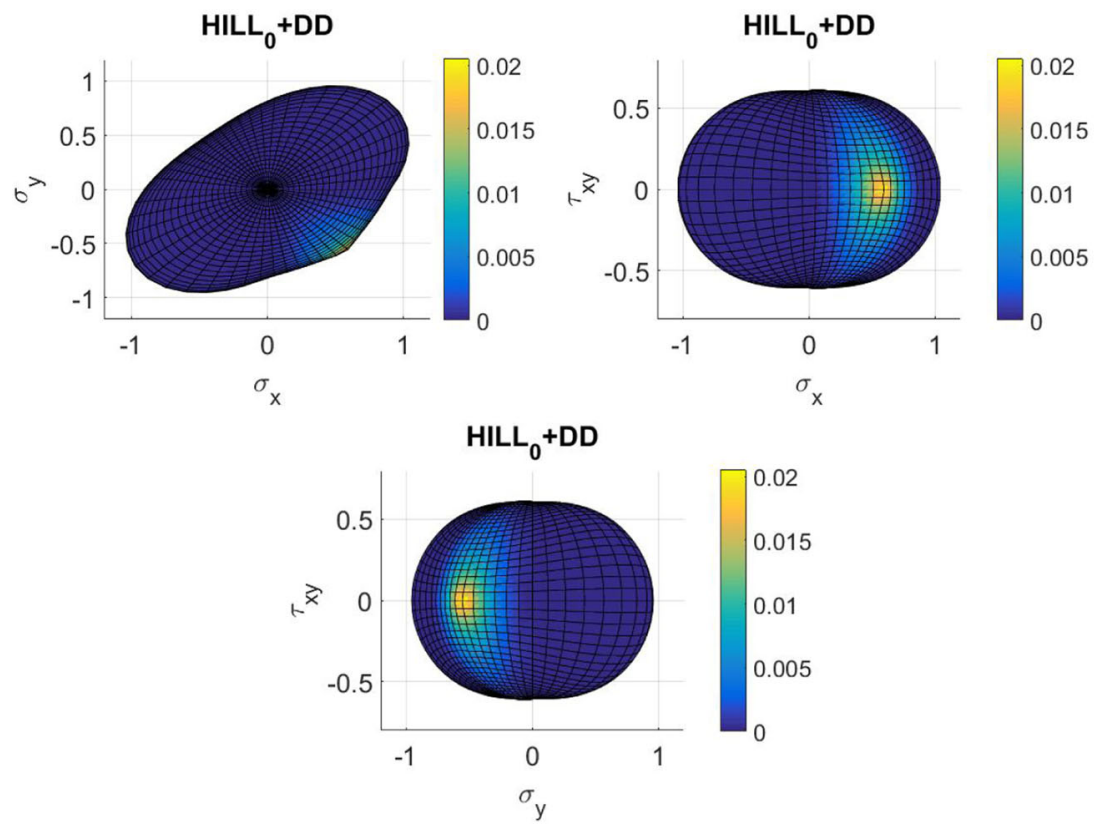

parametric space based on the existing set of experiments. Once the response surface is built, the global minimum of the error response surface will provide the point $c$ in the parametric space which is closest to reproduce the set of experiments.

Since the reference solution is known-it is provided by Barlat's model - it can be convenient to use it to check if our minimization problem in the kinematic variables also implies a good correction in the model. Certainly, this good correction is closely related with the good definition of the experiments.

At this point, several options could be adopted to reconstruct this response surface. Even though any nonstructured interpolation technique based on Delaunay triangularization can be used, it will suffer when the dimensionality of the parametric space increases. In this particular case, a non-linear sparse identification technique called Sparse Proper Generalized Decomposition (s-PGD) is used [25]. s-PGD strongly relies on the separation of variables to circumvent the problem of high dimensional spaces. Indeed its main objective is to capture the whole response surface using as few points as possible.

In brief, the s-PGD technique seeks to obtain a sparse regression of a parametric function-in our case, $E_{\boldsymbol{S}}(\boldsymbol{c})$ - by assuming a separate representation of the sought regression, say $\ell(\boldsymbol{c})$,

\section{Reconstruction of the error response surface by sparse sampling}

\section{Sparse approximation in high-dimensional spaces}

The objective of this procedure is to build a response surface for the error $E_{\mathcal{S}}(\boldsymbol{c})$ so as to characterize the
$\int_{\mathcal{I}} w^{*}(\boldsymbol{c})\left[\ell(\boldsymbol{c})-E_{s}(\boldsymbol{c})\right] d \boldsymbol{c}$,

where $\mathcal{I}$ represents the phase space in which $c$ evolves and $w^{*}$ a suitable test function. 
The main ingredient of the s-PGD technique relies on the assumption of a separate form for the sought approximation, i.e.,

$\ell(\boldsymbol{c}) \approx \ell^{M}(\boldsymbol{c})=\sum_{k=1}^{M} X_{1}^{k}\left(c_{1}\right) X_{2}^{k}\left(c_{2}\right) \cdots X_{\mathrm{n}_{\mathrm{param}}}^{k}\left(c_{\mathrm{n}_{\mathrm{param}}}\right)$.

This type of separate approximation has been tested in up to one hundred dimensions without any major difficulty, nor need of supercomputing facilities. The interested reader can consult [26] for more details.

In turn, the test function $w^{*}$ is chosen as formed by a sum of Dirac delta functions collocated at the sampling points,

$w^{*}(\boldsymbol{c})=\ell^{*}(\boldsymbol{c}) \sum_{i=1}^{P} \delta\left(\boldsymbol{c}_{i}\right)$,

where $\boldsymbol{c}_{i}$ represents one of the $P$ sampling points in the parametric space. Of course, if we are looking for a new term $k$ in the separated representation, the test function will look like

$$
\begin{aligned}
\ell^{*}(\boldsymbol{c})= & \left(X_{1}^{k}\right)^{*}\left(c_{1}\right) X_{2}^{k}\left(c_{2}\right) \cdots X_{\mathrm{n}_{\text {param }}^{k}}^{k}\left(c_{\mathrm{n}_{\text {param }}}\right) \\
& +X_{1}^{k}\left(c_{1}\right)\left(X_{2}^{k}\right)^{*}\left(c_{2}\right) \cdots X_{\mathrm{n}_{\text {param }}}\left(c_{\mathrm{n}_{\text {param }}}\right)+\ldots \\
& +X_{1}^{k}\left(c_{1}\right) X_{2}^{k}\left(c_{2}\right) \cdots\left(X_{\mathrm{n}_{\text {param }}}\right)^{*}\left(c_{\mathrm{n}_{\text {param }}}\right) .
\end{aligned}
$$

The precise form of the approximating functions $X_{j}^{k}\left(c_{j}\right)$ is found by resorting to a greedy algorithm followed by a fixed point linearization scheme, since we look for the precise form of products of functions, thus leading to a non-linear problem. To avoid Runge's phenomenon, that is, spurious oscillations in the approximated one-dimensional functions based on data-points that do not correspond to the Gauss-Lobatto-Chebyshev ones, interpolation based on kriging is retained.The interested reader will find every detail of the s-PGD methodology in [25]. Kriging possesses some interesting features. Since it is based on the fundamental assumption of data being Gaussian, it provides an easy filtering of noise, by giving the best linear unbiased prediction of the intermediate values.

\section{Line search in high-dimensional spaces}

The s-PGD algorithm provides a set of one-dimensional functions or, more commonly, modes which are able to reproduce a given function $\ell$ in the high dimensional space when they are combined as

$\ell\left(c_{1}, c_{2}, \ldots, c_{D}\right)=\sum_{k=1}^{M} \prod_{d=1}^{D} X_{d}^{k}\left(c_{d}\right)$.

Function $\ell$ is stored in a separated format, which has demonstrated to be very convenient in terms of memory consumption. However, sometimes the local extremes of $\ell$ are required, as in this case. Even if the simplest option could be to reconstruct the response surface $\ell\left(c_{1}, c_{2}, \ldots, c_{D}\right)$ in the high dimensional space, the memory requirements will increase exponentially with the dimensionality of the problem. In other words, if each mode is approximated by means of a finite element mesh of, say, 10 degrees of freedom, a problem defined in dimension $D$ implies to store $10^{D}$ nodal values.

In this case, we made an adaptation of the so-called line search minimization algorithm so that the consequent search directions coincide with the cartesian axes, thus exploiting the separated representation format.

Let us assume that the $i$-th dimension is going to be minimized. Consequently, the other coordinates are freezed at some value within their correspondent intervals of definition, i.e., $\hat{c}_{d}$ for $d \neq i$. By doing that, the problem reduces to a minimization problem in a one dimensional space:

$\hat{c}_{i}=\min _{c_{i}} \sum_{k=1}^{M}\left(\prod_{d \neq i}^{D} X_{d}^{k}\left(\hat{c}_{d}\right)\right) X_{i}^{k}\left(c_{i}\right)$.

When this minimization problem is finished, the search direction is updated to dimension $i=i+1$, repeating the same procedure. When the $\hat{c}_{d}$ coordinates do not change noticeably after one iteration for each dimension, the line search algorithm is finished.

To sum up the properties of the cartesian line search:

- There is no need to reconstruct the function $\ell\left(c_{1}, c_{2}, \ldots, c_{D}\right)$ in the high dimensional space, circumventing memory issues related to the storage.

- The global minimization problem is transformed into a set of one-dimensional minimization problems which are very efficient because all minimization directions coincide which the directions in which the solution has been separated.

- There is no guarantee that the obtained minimum is the global minimum of the function. In order to circumvent that problem, the algorithm is initialized at different starting positions, selecting the final point that presents the lower value of the function. This problem may appear in functions living in a high-dimensional space which do not have a certain level of regularity.

\section{Numerical results}

\section{Squared coupons}

\section{Test description}

Two different quadratic Hill criterions have been used as a starting point in our discrepancy model. The first one, $\mathcal{M}\left(\boldsymbol{p}_{1}\right)$, is defined by $F_{1}=2.1, G_{1}=1.8, H_{1}=0.7$ $N_{1}=1.9$, while the second one, $\mathcal{M}\left(\boldsymbol{p}_{2}\right)$ is defined by $F_{2}=2.3, G_{2}=2.0, H_{2}=0.8 N_{2}=1.7$. While 
Fig. 4 Different views of $\mathcal{M}\left(\boldsymbol{p}_{1}\right)$ plastic yield function. The color map represents the mismatch between the $\mathcal{M}\left(\boldsymbol{p}_{1}\right)$ criterion and the Barlat Yld2004-18p, and thus $\mathcal{R}-\mathcal{M}\left(\boldsymbol{p}_{1}\right)=\mathcal{D}$, the objective function to be captured by our discrepancy model.

$E_{\mathcal{F}_{Y}}\left(\mathcal{M}\left(\boldsymbol{p}_{1}\right)\right)=1.57$
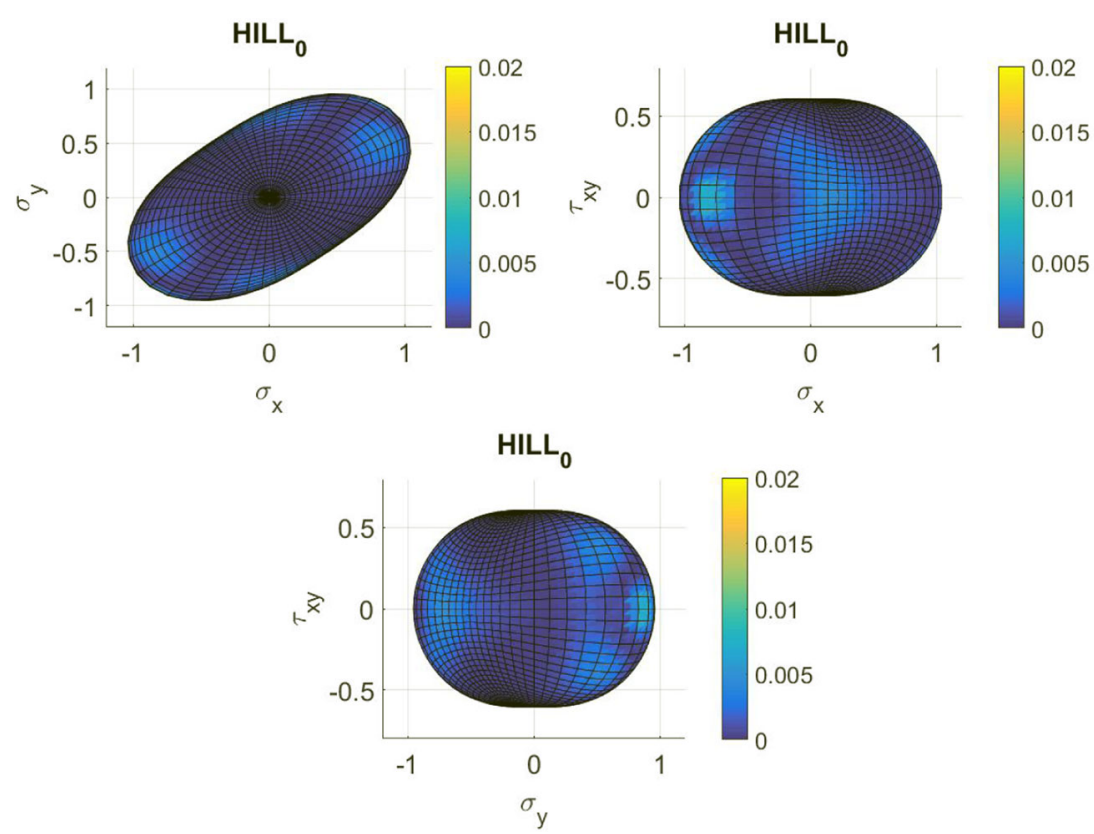

$\mathcal{M}\left(\boldsymbol{p}_{1}\right)$ is already quite close to Barlat's criterion, with an error $E_{\mathcal{F}_{Y}}\left(\mathcal{M}\left(\boldsymbol{p}_{1}\right)\right)=1.57$, the second one presents an error $E_{\mathcal{F}_{Y}}\left(\mathcal{M}\left(\boldsymbol{p}_{2}\right)\right)=24.9$. This could correspond, so to speak, to the case of a poorly calibrated poor model. Figure 4 depicts the point-wise difference between the $\mathcal{M}\left(\boldsymbol{p}_{1}\right)$ criterion and the Barlat Yld2004-18p projected on the $\mathcal{M}\left(\boldsymbol{p}_{1}\right)$ surface. Figure 5, in turn, presents the same error for the model $\mathcal{M}\left(\boldsymbol{p}_{2}\right)$.

Regarding the set of experiments $s$ necessary to calibrate the discrepancy model, we have chosen to use a set of simple tests in a coupon of size $\Omega=[0,1] \times[0,1]$. These will be defined by the following set of boundary conditions,

$$
\begin{gathered}
u_{x}(0, y)=0, \\
u_{y}(x, 0)=0, \\
\boldsymbol{\sigma}(1, y) \boldsymbol{n}=\boldsymbol{t}_{1}, \\
\boldsymbol{\sigma}(x, 1) \boldsymbol{n}=\boldsymbol{t}_{2} .
\end{gathered}
$$

Hence, varying both tractions $\boldsymbol{t}_{1}$ and $\boldsymbol{t}_{2}$, different regions of the stress space inside the coupon are explored. Indeed, 40
Fig. 5 Different views of $\mathcal{M}\left(\boldsymbol{p}_{2}\right)$ plastic yield function. The color map represents the point-wise difference between the $\mathcal{M}\left(\boldsymbol{p}_{2}\right)$ criterion and the Barlat Yld2004-18p. $E_{\mathcal{F}_{Y}}\left(\mathcal{M}\left(\boldsymbol{p}_{2}\right)\right)=24.9$
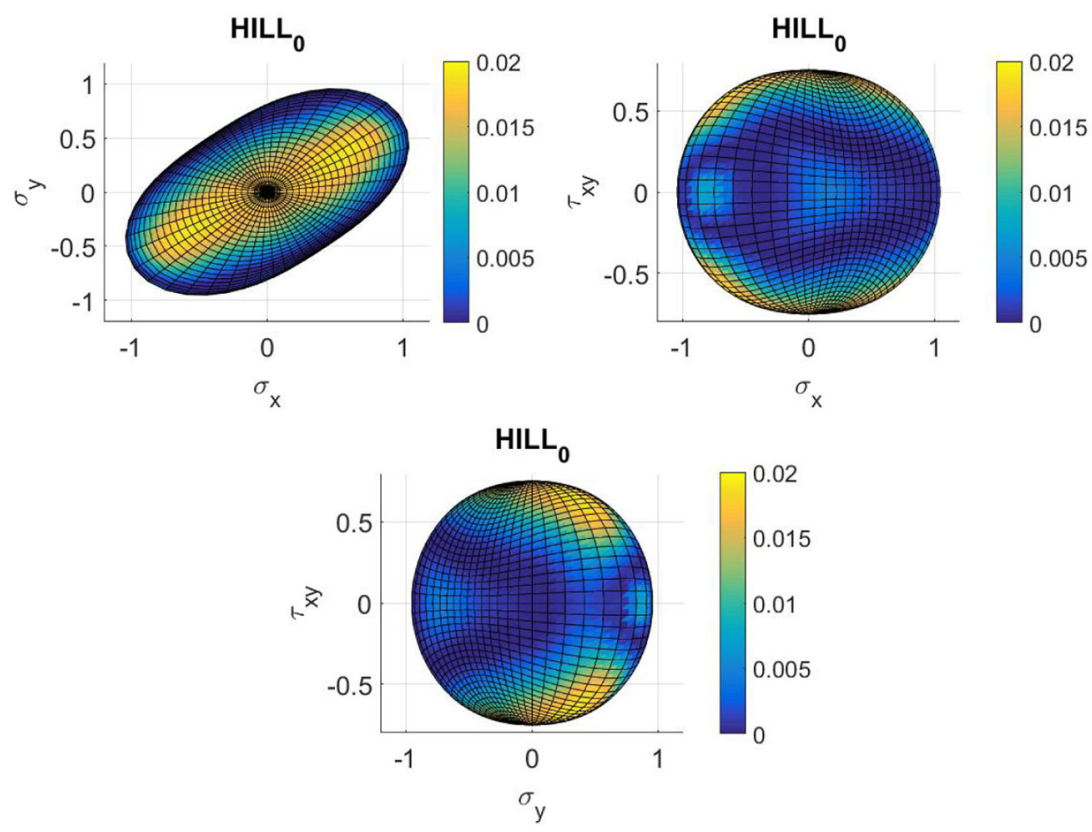


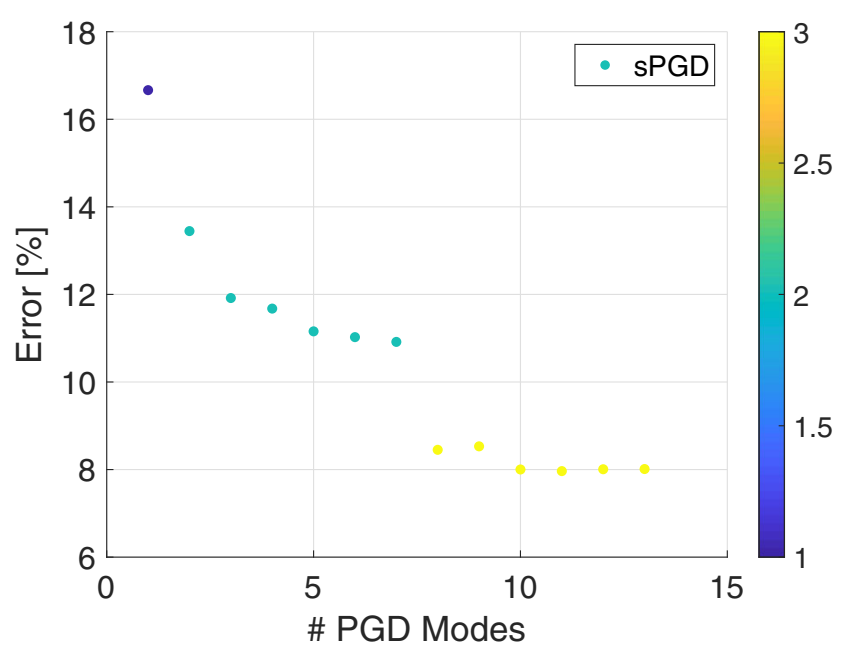

Fig. 6 Error in the reconstruction $E_{\boldsymbol{s}}\left(\mathcal{M}\left(\boldsymbol{p}_{1}\right)+\mathcal{D} ; \boldsymbol{c}\right)$ using half of the points in the data base for training

different experiments have been included in order to create $E_{s}(\boldsymbol{c})$, so that $|\boldsymbol{s}|=40$.

\section{Construction of the error response surfaces}

In order to build the response surfaces $E_{s}\left(\mathcal{M}\left(\boldsymbol{p}_{1}\right) ; \boldsymbol{c}\right)$ and $E_{s}\left(\mathcal{M}\left(\boldsymbol{p}_{2}\right) ; \boldsymbol{c}\right), 1000$ simulations, randomly sampling the parametric space $c$, have been accomplished for each model $\mathcal{M}\left(\boldsymbol{p}_{1}\right)$ and $\mathcal{M}\left(\boldsymbol{p}_{2}\right)$. Each realization in the parametric space follows a uniform distribution from $[-0.1,0.1]$ in the $\mathcal{M}\left(\boldsymbol{p}_{1}\right)$ case and $[-0.15,0.15]$ in the $\mathcal{M}\left(\boldsymbol{p}_{2}\right)$ case, since we expect the need for a major correction.

As can be noticed in Fig. 6 the obtained response surface for $\mathcal{M}\left(\boldsymbol{p}_{1}\right)$ presents around $8 \%$ of mean relative error. This error could be decreased easily if more sample points are added to the s-PGD algorithm.

\section{Error minimization and obtention of the sought correction}

Once the response surface has a continuous and separated representation, the minimum is searched by employing a line search in each one of the separated directions. The initial point at which the line search algorithm is started is changed randomly to ensure the global character of the minimum.

Figure 7 shows the error when the yield surface $\mathcal{M}\left(\boldsymbol{p}_{1}\right)$ is corrected with the obtained discrepancy model. Note that the final reconstructed error $E_{\mathcal{F}_{Y}}\left(\mathcal{M}\left(\boldsymbol{p}_{1}\right)+\mathcal{D}\right)$, has been reduced with respect to the $E_{\mathcal{F}_{Y}}\left(\mathcal{M}\left(\boldsymbol{p}_{1}\right)\right)$ error, passing from 1.57 to 1.27 . This is equivalent to a $19 \%$ of improvement thanks to the data driven correction for this particular case.

Figure 8 shows the error when the yield surface $\mathcal{M}\left(\boldsymbol{p}_{2}\right)$ is improved with the data driven correction. In this particular case, the final reconstructed error $E_{\mathcal{F}_{Y}}\left(\mathcal{M}\left(\boldsymbol{p}_{2}\right)+\mathcal{D}\right)$ has been reduced as well from 24.9 to 4.63 . Therefore, some $81 \%$ of improvement has been obtained in this particular case.

\section{Coupon subject to bending loads}

In this example a bar with both ends clamped, in which a uniform vertical negative distributed load is acting along both top and bottom sides.

Figure 9 (top) shows the cumulated strain error between Barlat's and Hill's $\mathcal{M}\left(\boldsymbol{p}_{2}\right)$ yield functions. The bottom
Fig. 7 Different views of $\mathcal{M}\left(\boldsymbol{p}_{1}\right)+\mathcal{D}$ plastic yield function. The color map represents the mismatch between the $\mathcal{M}\left(\boldsymbol{p}_{1}\right)+\mathcal{D}$ criterion and the Barlat Yld2004-18p. $E_{\mathcal{F}_{Y}}\left(\mathcal{M}\left(p_{1}\right)+\mathcal{D}\right)=1.27$
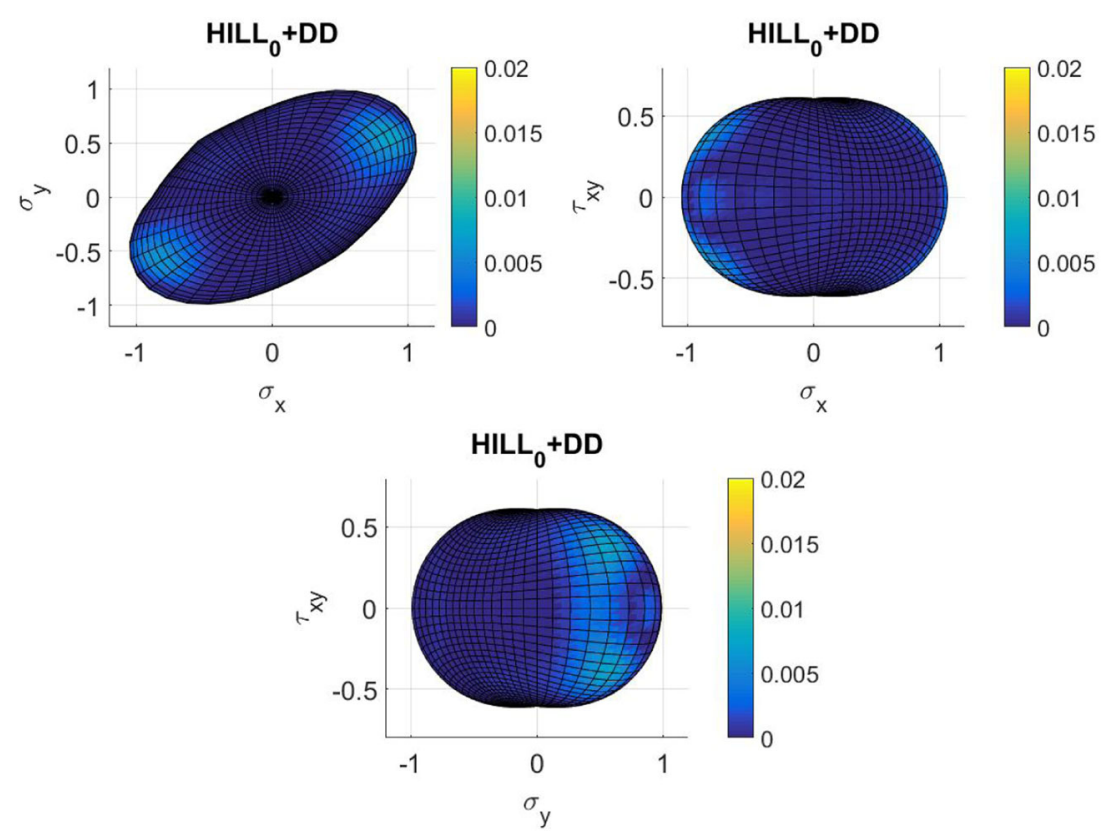
Fig. 8 Different views of $\mathcal{M}\left(\boldsymbol{p}_{2}\right)+\mathcal{D}$ plastic yield function. The color map represents the mismatch between the $\mathcal{M}\left(\boldsymbol{p}_{2}\right)+\mathcal{D}$ criterion and the Barlat Yld2004-18p. $E_{\mathcal{F}_{Y}}\left(\mathcal{M}\left(p_{2}\right)+\mathcal{D}\right)=4.63$
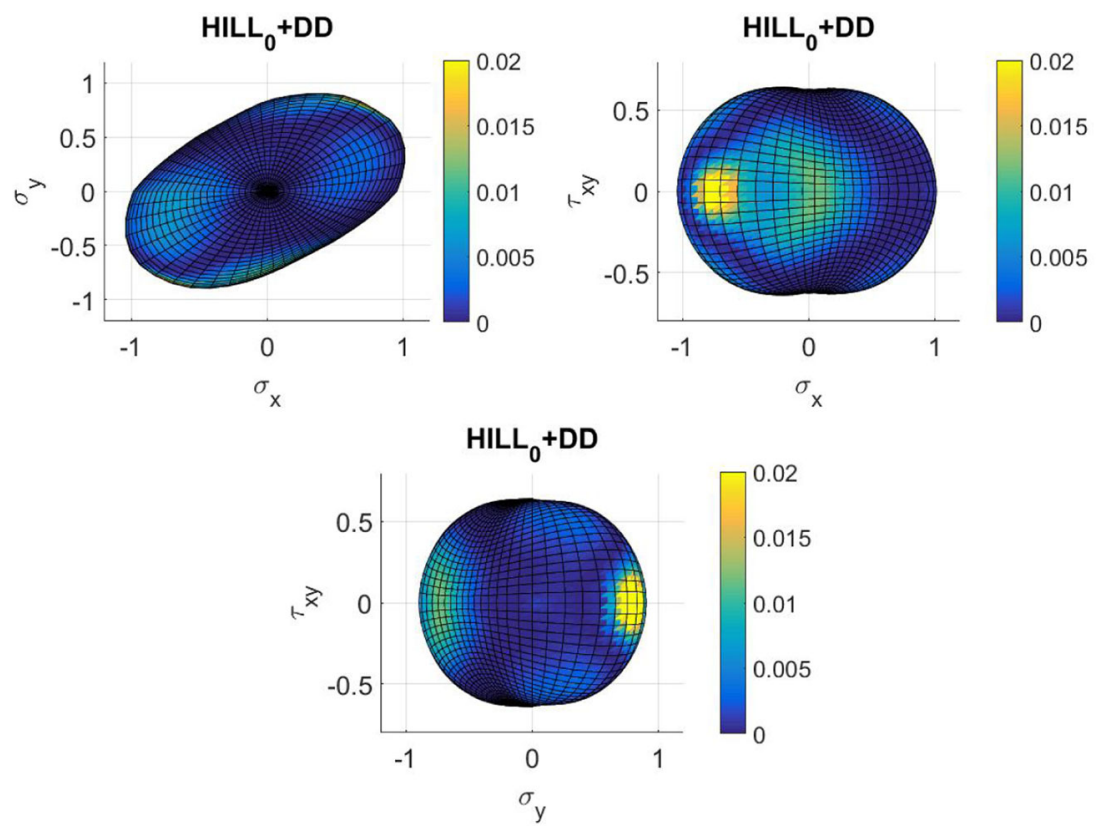

\section{Conclusions}

Hill's yield functions. As it can be noticed, the error in the strain field is reduced when considering the correction. However, this error does not vanish, since the correction does not reproduce perfectly well Barlat's criterion for the considered sampling. It asymptotically decreases, however, when more data points are considered.

In light of the results, the importance of data driven corrections is higher when the model is less accurate, since few data produce important improvement.
In recent times a lot of attention has been paid to the development of machine learning techniques able to unveil governing equations from data. This is specially important for constitutive equations that, unlike other more epistemologically sound equations-like equilibrium, for instance-are often phenomenological and inexact. Their precise expression is found by data fitting, leading very often to poor fitting to the experimental results.
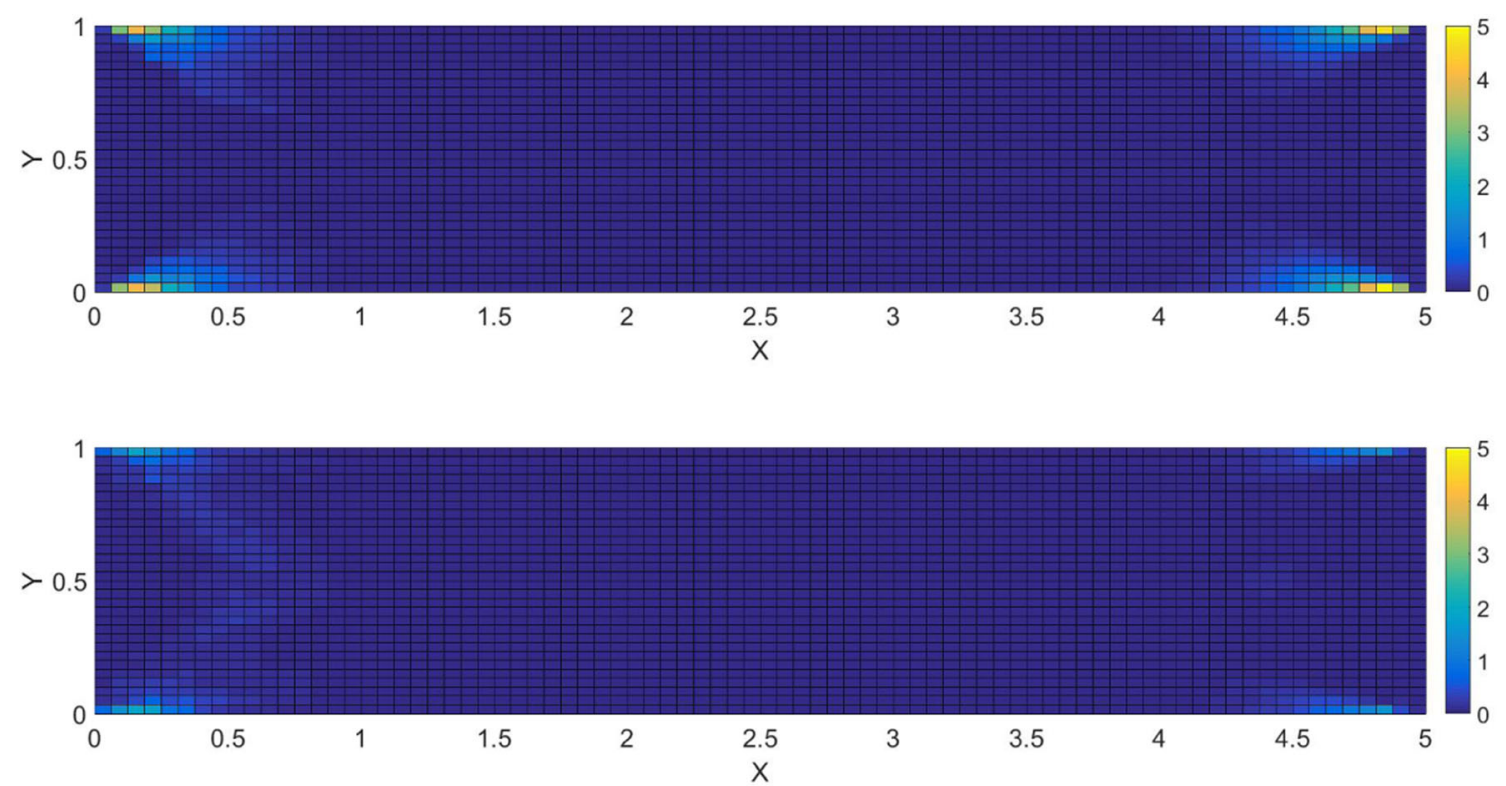

Fig. 9 Cumulated strain error between Barlat's and Hill's models (top) and between Barlat's and Hill's plus data correction (bottom) 
Unlike previous approaches, we believe that it is important not to discard all the existing knowledge concerning constitutive equations (particularly plastic yield functions, for which an extensive corps of literature exists). Instead of learning models from scratch, we believe that it will be much more efficient and appealing to try to correct existing models in light of the obtained experimental results and the observed discrepancies.

In this paper we have developed a method for the correction of plasticity models with the help of experimental data that makes use of sparse identification techniques in high-dimensional spaces. Particularly, we employ the sparse-PGD method [25], that has rendered excellent results for the examples considered herein. In order to circumvent the problem of approximating a function in the high dimensional space, it is important to make use of adequate interpolation techniques, which are able to provide us with a reasonable estimation of the response surface.

Noteworthy, results obtained numerically from a Barlat Yld2004-18p yield function were approximated by assuming a Hill model and then obtaining, in a completely automated fashion, a suitable correction.

The presented method paves the way for the development of a completely general, hybrid constitutive modeling methodology for the obtaining of accurate constitutive models by summing up the best of both worlds: all the experience accumulated in the last century with constitutive modeling of solids and the best of machine learning.

Acknowledgements This project has received funding from the European Union's Horizon 2020 research and innovation program under the Marie Sklodowska-Curie grant agreement No. 675919. Also by the Spanish Ministry of Economy and Competitiveness through Grants number DPI2017-85139-C2-1-R and DPI2015-72365-EXP and by the Regional Government of Aragon and the European Social Fund, research group T24 17R.

\section{References}

1. Ogden RW (1972) Large deformation isotropic elasticity - on the correlation of theory and experiment for incompressible rubberlike solids. Proc R Soc London Math Phys Eng Sci 326(1567):565-584

2. Arruda EM, Boyce MC (1993) A three-dimensional constitutive model for the large stretch behavior of rubber elastic materials. J Mech Phys Solids 41(2):389-412

3. Holzapfel GA, Gasser TC (2000) A new constitutive framework for arterial wall mechanics and a comparative study of material models. J Elast 61:1-48

4. Simo JC, Hughes TJR (2006) Computational inelasticity, vol 7. Springer Science \& Business Media

5. Hill R (1948) A theory of the yielding and plastic flow of anisotropic metals. Proc R Soc London A Math Phys Eng Sci 193(1033):281-297
6. Kirchdoerfer T, Ortiz M (2016) Data-driven computational mechanics. Comput Methods Appl Mech Eng 304:81-101

7. Kirchdoerfer T, Ortiz M (2017) Data driven computing with noisy material data sets. Comput Methods Appl Mech Eng 326:622-641

8. González D, Aguado JV, Cueto E, Abisset-Chavanne E, Chinesta F (2018) kpca-based parametric solutions within the pgd framework. Arch Comput Methods Eng 25(1):69-86

9. Lopez E, Gonzalez D, Aguado JV, Abisset-Chavanne E, Cueto E, Binetruy C, Chinesta F (2018) A manifold learning approach for integrated computational materials engineering. Arch Comput Methods Eng 25(1):59-68

10. Ibanez R, Abisset-Chavanne E, Aguado JV, Gonzalez D, Cueto E, Chinesta F (2018) A manifold learning approach to data-driven computational elasticity and inelasticity. Arch Comput Methods Eng 25(1):47-57

11. Ibanez R, Borzacchiello D, Aguado JV, Abisset-Chavanne E, Cueto E, Ladeveze P, Chinesta F (2017) Data-driven nonlinear elasticity: constitutive manifold construction and problem discretization. Comput Mech 60(5):813-826

12. González D, Chinesta F, Cueto E (May 2018) Thermodynamically consistent data-driven computational mechanics. Continuum Mechanics and Thermodynamics. https://doi.org/10.1007/s00161018-0677-z

13. Bessa MA, Bostanabad R, Liu Z, Hu A, Apley DW, Brinson C, Chen W, Liu WK (2017) A framework for data-driven analysis of materials under uncertainty: countering the curse of dimensionality. Comput Methods Appl Mech Eng 320:633-667

14. Crespo J, Latorre M, Montáns FJ (2017) Wypiwyg hyperelasticity for isotropic, compressible materials. Comput Mech 59(1):73-92

15. De Rosa E, Latorre M, Montáns FJ (2017) Capturing anisotropic constitutive models with wypiwyg hyperelasticity; and on consistency with the infinitesimal theory at all deformation levels. Int J Non-Linear Mech 96:75-92

16. Brunton SL, Proctor JL, Kutz JN (2016) Discovering governing equations from data by sparse identification of nonlinear dynamical systems. Proc Nat Acad Sci USA 113(15):3932-3937

17. Mangan NM, Brunton SL, Proctor JL, Kutz JN (2016) Inferring biological networks by sparse identification of nonlinear dynamics. IEEE Trans Molec Biol Multi-Scale Commun 2(1):52-63

18. Quade M, Abel M, Kutz JN, Brunton SL (2018) Sparse identification of nonlinear dynamics for rapid model recovery. Chaos: Interdiscip J Nonlinear Sci 28(6):063116

19. Raissi M, Perdikaris P, Karniadakis GE (2017) Machine learning of linear differential equations using gaussian processes. J Comput Phys 348:683-693

20. Raissi M, Karniadakis GE (2018) Hidden physics models: machine learning of nonlinear partial differential equations. J Comput Phys 357:125-141

21. Raissi M, Perdikaris P, Karniadakis GE (2017) Inferring solutions of differential equations using noisy multi-fidelity data. J Comput Phys 335:736-746

22. Yoon JW, Barlat F, Dick RE, Karabin ME (2006) Prediction of six or eight ears in a drawn cup based on a new anisotropic yield function. Int J Plast 22(1):174-193

23. Barlat F, Aretz H, Yoon JW, Karabin ME, Brem JC, Dick RE (2005) Linear transfomation-based anisotropic yield functions. Int J Plast 21(5):1009-1039

24. Sibson R (1981) A brief description of natural neighbour interpolation. In: Barnett $\mathrm{V}$ (ed) Interpreting multivariate data. Wiley, pp 21-36

25. Ibanez R, Abisset-Chavanne E, Ammar A, Gonzalez D, Cueto E, Huerta A, Duval JL, Chinesta F (2018) A multi-dimensional data-driven sparse identification technique: the sparse proper generalized decomposition. Complexity, In press

26. Chinesta F, Cueto E (2014) PGD-based modeling of materials structures and processes. Springer International Publishing, Switzerland 